\title{
Geometry of numbers and dimensionality
}

\author{
Subhash Kak \\ Oklahoma State University, Stillwater \\ Email: subhash.kak@okstate.edu \\ August 10, 2020
}

\begin{abstract}
The paper presents an observer-centric analysis of optimal representation that helps define certain relationship between geometry of numbers and corresponding dimensionality. The motivation for this research is the complementarity that exists in looking at numerical data by itself as compared to its description within a pre-supposed structural framework. Specifically, we consider estimates based on intrinsic dimensionality of data and compare these to those where an a priori order is imposed on the data. We show that this approach helps solve the long-standing problem of different values of the Hubble constant obtained using two different methods.
\end{abstract}

\section{Introduction}

Scholars have long debated whether numbers are more fundamental than axiomatic geometry. If one takes geometry to be primary, one can fit in numbers to describe location of objects and their separation. If one takes integers to be more fundamental, then one is led first to negative numbers, then to rational and irrational numbers, and further to complex numbers that have an elegant geometric interpretation. Geometry and numbers can be taken to represent complementary sides of the same reality. There is a meeting of the two in geometry of numbers, algebraic geometry, and differentiable and analytic manifolds.

In traditional geometry, one begins with notions of point, plane, and the threedimensional space. One also speaks of higher dimensions in the configuration space of a physical system, with dimensions equal to the system's degrees of freedom. In general topology, the concept of dimension is extended from natural numbers, to infinite dimensions such as Hilbert spaces and positive real numbers in fractal geometry.

Complementary descriptions and ways of bridging them have long provided insights into physical phenomena. A most striking pair of complementary descriptions is that of physical reality as collection of things, $\Psi$, in space and as change, $\frac{d \Psi}{d t}$, in these things. One of the key results of quantum theory was that the two were equivalent descriptions related through a constant of proportionality: 


$$
i \hbar \frac{d \Psi(\mathrm{t})}{d t}=\widehat{H} \Psi(\mathrm{t})
$$

where $\hbar$ is the reduced Planck's constant, $\Psi(\mathrm{t})$ is the state function, and $\widehat{H}$ is the Hamiltonian operator.

Going back to the beginnings of modern physics, space and time were viewed as separate categories. In Newton's Principia, which formulated the classical picture of reality, the understanding of time, space, place, and motion is presented in two ways: first, in the relative conception of them, and, second, in the absolute, true, mathematical quantities themselves. Newton speaks of time and space thus [1]: "Absolute, true, and mathematical time, from its own nature, passes equably without relation to anything external, and thus without reference to any change or way of measuring of time (e.g., the hour, day, month, or year)"; and "Absolute, true, and mathematical space remains similar and immovable without relation to anything external." Absolute time and space were not defined, but motion of material objects was ultimately conceived with respect to these.

Amongst pre-modern synthesizers, the views of Kaṇāda, the author of the Vaiśeșika Sūtra, who speaks of nine classes of substances, some of which are non-atomic, some atomic, and others all-pervasive, are noteworthy [2]. The non-atomic ground is provided by the three substances of ether, space, and time, which are unitary and indestructible; a further four are composed of indivisible, and indestructible atoms; in addition, there are categories of consciousness and the mind. In this view, time and space are relative in relation to the observer. It is an amusing sidelight to history of physics that these ideas influenced Nicola Tesla [3] and through him may have also influenced mainstream physics of early twentieth century. In general, this position is in consonance with the notion that mathematical objects are a creation of the mind, and not independent abstractions in themselves.

Newton's rival, Leibniz proposed a different view of time in which events are more fundamental than time instants. According to Leibniz, the primary role is to be ascribed to matter and its properties and it is in relation to these properties that one can speak of time and space and not in any absolute sense. Time is simply succession of instantaneous configurations of matter and not something that flows independent of the bodies in the universe [4]. Mach developed Leibniz's ideas further by postulating that only such concepts should be used in physics that have an empirical basis. This eventually led to the theory of relativity and the notion of spacetime. 
Another complementarity is between information and structure. There is yet no resolution of the measurement problem of quantum mechanics in which the process of observation leads to the collapse of the wave function (that otherwise evolves deterministically) which then becomes the origin of the information obtained by the observer. To go around this difficulty, it has been proposed that binary information (bits) itself is fundamental to our understanding of reality. This was expressed by Wheeler in his declaration It from bit suggesting that binary information is the ground stuff of physical reality [5]. The complementarity then is between the information obtained by the observer and its relationship with the object's structure.

With this as background, we wish to enlarge the question to beyond the response to questions that elicit yes-no (i.e. binary) responses. Contra Wheeler, we argue using information theory that it is incorrect to assume that information obtained from Nature is fundamentally binary. We take up the question of optimum representation of numerical information and its possible relationship to the dimensionality of the data.

Specifically, we show that considerations of information and mathematical logic require that the optimal dimension to be associated with the physical space is $e=2.71828$..., and not 3. If physical reality is e-dimensional and we insist on viewing it as 3-dimensional then there is a discrepancy equal to $e / 3=0.9060$; this number is very close to the divergence of $67 / 74=0.9054$ in the experimental data of the Hubble constant obtained using two different methods.

\section{Information and dimensions}

If we go along with Wheeler and consider the bit as the building block of reality, we can map binary sequences to locations in space. Thus, for example, binary sequences 000 , $001, \ldots, 111$, may be mapped to the corners of a 3 -dimensional cube (Figure 1). Indeed, such mapping of binary strings to locations in space is a factor in the intuition that binary information represents the fundamental building-block of reality.

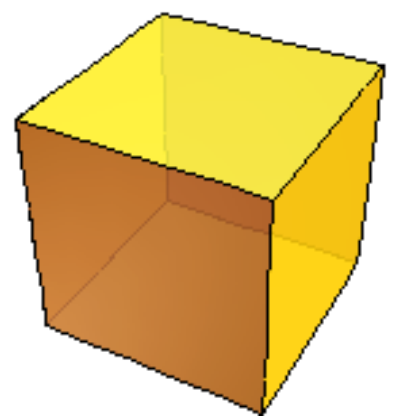

Figure 1. A cube where the corners are binary sequences 000, 001, .. 111 
But there is circularity in this above argument. Binary implies exclusion in relation to the underlying question which means that the location in space should be definable in terms of three exclusive dimensions. In other words, binary strings implicitly presuppose Euclidean space (which is later modified in general theory of relativity). Further this ontology leaves out the observer of reality, and, therefore, it could not be complete, which is reminiscent of similar objections to completeness of theory in quantum mechanics [6].

The assumption that space is three-dimensional Euclidean is the Platonic position of mathematical philosophy according to which mathematical concepts have an independent reality. But as we know it is necessary to examine all underlying assumptions in formal systems to see if they square with our intuition, which, in itself, must be tested against experimental observations.

The measure of information is related to frequency of the event: the information is more the less likely the event. The logarithm measure associated with information has become widely accepted because it is additive. In other words, additivity of information is the underlying unstated assumption in the information-theoretic approach to structure.

A mapping that is more efficient than binary is ternary, although it is also not optimal. Attention was given by logicians and computer scientists in the 60 s and 70 s to build computers based on ternary logic [7], which would be more efficient than computers built on binary logic. But for a variety of complicated engineering reasons, such as simpler and more reliable binary gates could be implemented then using available technology, those efforts were not successful and binary logic machines eventually became entrenched. Nevertheless, in tasks where the possibilities must be an integer, ternary logic is superior to binary logic [8].

I have recently argued [9][10] that based on information theory we are compelled to accept that space is e-dimensional and not 3-dimensional. I recommend the original papers to the reader but the main argument will be summarized in the next section. It appears that since $e$ is quite close to 3 , and since it is easy to visualize integer dimensions, our intuition takes space to be 3-dimensional.

\section{Optimal number of dimensions}

The mathematical argument is the e-dimensions are the most efficient representation of information and since Nature chooses optimality, as in physical laws such as the Principle of Least Action, the actual physical space should have this dimensionality. 
Although it is troublesome to visualize non-integer and irrational number of dimensions, formal analysis of such systems has been done [11][12]. Indeed, irrational number of dimensions provide explanation for recursion and scale-invariance in complex systems -- biological, physical, or engineered - and explain fractal behavior [9][10][13], examples of which include the Mandelbrot set, patterns in Romanesco broccoli, snowflakes, the Nautilus shell, complex computer networks, brain structure, as well as the filament structure and distribution of matter in cosmology.

If space were $d$-dimensional, one could label the dimensions as $1,2,3, \ldots d$. The probability of the use of each of the $d$ dimensions may, from considerations related to maximization of entropy, be taken to be the same and equal to $1 / d$. Therefore, the information associated with each dimension is $\ln d$.

Clearly, this information increases as $d$ increases. But this increase must be balanced against the cost of the use of the larger dimension set. Information efficiency per dimension is:

$$
E(d)=\frac{\ln d}{d}
$$

Its maximum value is obtained by taking the derivative of $E(d)$ and equating that to zero, which yields $d_{\text {optimum }}=e=2.71828 \ldots$. In other words:

Theorem. The optimal number of dimensions associated with space is $e$.

Table 1 gives the value of $E(d)$ in bits (where the measure is $\frac{\log _{2} d}{d}$ ) for $d$ ranging from 2 to 10 , together with the additional value for the optimum $d=e$.

Table 1. Efficiency of space of dimensions ranging from 2 to 10

\begin{tabular}{|l|l|l|l|l|l|l|l|}
\hline$d$ & 2 & $\mathrm{e}$ & 3 & 4 & 5 & 8 & 10 \\
\hline$E(d)$ bits & 0.500 & 0.531 & 0.528 & 0.500 & 0.465 & 0.375 & 0.331 \\
\hline
\end{tabular}

We may conclude that Nature doesn't count in $2 \mathrm{~s}, 3 \mathrm{~s}$, or $10 \mathrm{~s}$. The optimal base value for representation of numbers is e $=2.718$, and the integer closest to it, namely 3 , is more efficient than binary. The superiority of $e$-dimensions in terms of efficiency is evident in Figure 2. 


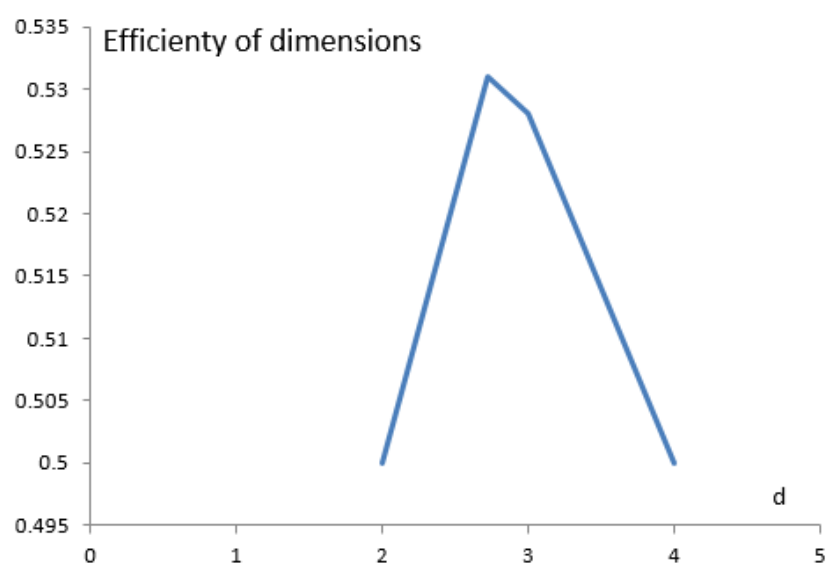

Figure 2. Efficiency of dimensions for $\mathrm{d}=2, e, 3$, and 4

One can set up experimental tests to determine dimensionality of space. For one, the geodesics associated with an e-dimensional space would be different from the Euclidean 3-dimensional space. Space may be locally 3-dimensional but globally depart from it to $e$-dimensionality. One can set up experiments such as cube packing or sphere packing. In case of cube packing, one would be unable to pack 27 cubes of $1 / 3$ the size of the larger cube for as we saw in [10], the number of small cubes that fits in the larger cube is smaller and we see this most vividly in the fractal approximations to $e$-space. Likewise, the number of smaller cubes that may be fitted into a larger cube will be less for an e-dimensional space compared to the 3-dimensional space. But accumulated errors in shape and size measurements that vary with temperature and body stresses would limit the precision with which the actual physical dimension can be determined.

A non-integer, irrational number for dimensionality has implications not only in study of complex system behavior (as in [9]) but also for aerospace engineering, as in improving spacecraft navigation and trajectory data [14], as well in physics [10] and the study of cosmology. I do not wish to revisit the implications already discussed in the previous papers, but here I consider relevance to a much-discussed problem related to estimates of the Hubble constant.

\section{Two different estimates of the Hubble constant}

There are two conflicting values of the Hubble constant $\left(H_{0}\right)$ based on whether one analyzes the cosmic microwave background (CMB) (the "early" universe estimate) or observes motions of stars and galaxies (the "late" universe estimate). According to the Planck Collaboration [15] exploring the early universe, $H_{0}$ is about $67 \mathrm{~km} \mathrm{~s}^{-1} \mathrm{Mpc}^{-1}$, whereas a late universe estimate, Supernova $\mathrm{H}_{0}$ for the Equation of State (SHoES), has it at about $74 \mathrm{~km} \mathrm{~s}^{-1} \mathrm{Mpc}^{-1}[16]$. 
Planck's estimate of $H_{0}$ relies on measuring features in the CMB using Lambda-CDM, the standard model of cosmology. On the other hand, SHoES estimates the universe's expansion rate by measuring distances to other galaxies from the supernovas' distance and redshift, where brightness is related to luminosity by calibration against standard candles, which for SHoES are the Cepheid variables.

The independent Megamaser Cosmology Project considers galaxies with disks of waterbearing molecular gas orbiting supermassive black holes at galaxy centers. Considering distance measurements to four galaxies, and combined with previous distance measurements of two other galaxies, the calculations produce a value for $H_{0}$ of $73.9 \mathrm{~km}$ $\mathrm{s}^{-1} \mathrm{Mpc}^{-1}$ [17] that is nearly identical to the SHoES estimate. The discrepancy between the early and late universe estimates of the Hubble constant has not yielded to any analysis and it has been termed a crisis in physics [18].

We now present a resolution to the problem of the diverging estimates of the Hubble constant $\mathrm{H}_{0}$, based on early- or late- universe models. The discrepancy is between the values of $67 \mathrm{~km} \mathrm{~s}^{-1} \mathrm{Mpc}^{-1}$ from the early universe and $74 \mathrm{~km} \mathrm{~s}^{-1} \mathrm{Mpc}^{-1}$ from the late universe. This resolution comes from an information-theoretic perspective that implies that the optimal dimension to be associated with physical space is $e=2.71828$. If physical reality is e-dimensional and we insist on viewing it as being 3-dimensional then

there is a discrepancy equal to $\frac{e}{3}=0.9060$. This number is very close to the divergence of $\frac{67}{74}=0.9054$ from the experimental data.

The three-dimensional space is off from the e-dimensional optimal space by about 0.003 , or about 0.6 percent. One may speculate that since our cognitions are based on counting, we associate the nearest integer space of 3 dimensions to space which leads to our normal sense about its nature.

\section{Conclusions}

The paper presented arguments on the relationship between geometry of numbers and corresponding dimensionality. A recent result from information theory that data from physical space has an optimal dimensionality of $e=2.71828$ was advanced as the basis of the assumption that physical space has the same dimensionality.

As confirmation of this idea, we considered the problem of the discrepancy of the Hubble constant based on early and late universe models and showed that this discrepancy disappears if one recognizes that the early universe model provides the true $e$-dimensional estimate whereas the late universe model imposes a 3-dimensional 
gloss on our measurement that arises from the mathematical models that are fitted into the actual measurements.

\section{REFERENCES}

1. I. Newton, Philosophiae Naturalis Principia Mathematica, Bk. 1, 1689; Florian Cajori (ed.), Berkeley: University of California Press (1934)

2. S. Kak, Matter and Mind. Mount Meru Publishing (2016)

3. S. Kak, Tesla, wireless energy transmission and Vivekananda. Current Science 113: 2207-2209 (2017)

4. R. Arthur, Space and Relativity in Newton and Leibniz. British Journal for the Philosophy of Science, 45(1): 219-240 (1994)

5. J.A. Wheeler, A Journey into Gravity and Spacetime. W.H. Freeman (1990)

6. S. Kak, Epistemic view of quantum communication. In Quantum Foundations, Probability and Information, A. Khrennikov and B. Toni (editors). Springer-Verlag International, 119-128 (2018)

7. B. Hayes, Third base. Am. Sci. 89, 490-494 (2001)

8. S. Kak, On ternary coding and three-valued logic. arXiv:1807.06419 (2018)

9. S. Kak, The base-e representation of numbers and the power law. Circuits Syst. Signal Process. (2020); arXiv:2003.02345 (2020)

10. S. Kak, Information, representation, and structure. International Conference on Recent Trends in Mathematics and Its Applications to Graphs, Networks and Petri Nets, New Delhi, July 20-25 (2020)

11. F.H. Stillinger, Axiomatic basis for spaces with noninteger dimensions. Journal of Mathematical Physics, 18: 1224- 1234 (1977).

12. P. Mattila, Geometry of Sets and Measures in Euclidean Space: Fractals and Rectifiability. Cambridge University Press (1999)

13. K. Falconer, Fractals. Oxford University Press (2013)

14. L. Acedo, P. Piqueras and J. A. Morano, A possible flyby anomaly for Juno at Jupiter. arXiv:1711.08893 (2017)

15. Planck Collaboration. Planck 2018 results. VI. Cosmological parameters. Astronomy \& Astrophysics, July 16 (2018)

16. A.G. Riess, S. Casertano, W. Yuan, L. M. Macri, D. Scolnic, Large Magellanic Cloud Cepheid Standards Provide a 1\% Foundation for the Determination of the Hubble Constant and Stronger Evidence for Physics Beyond LambdaCDM. arXiv:1903.07603 (2019)

17. D. W. Pesce, J. A. Braatz, M. J. Reid, A. G. Riess, D. Scolnic, J. J. Condon, F. Gao, C. Henkel, C. M. V. Impellizzeri, C. Y. Kuo, K. Y. Lo. The Megamaser Cosmology Project. XIII. Combined Hubble Constant Constraints. The Astrophysical Journal, 891 (1): L1 (2020)

18. R. Panek, A Cosmic crisis. Scientific American 322, 3, 30-37 (March 2020) 